\title{
Comparison of yield and relative costs of different screening algorithms for tuberculosis in active case-finding: a cross-section study
}

Fei Zhao 1,2,3, Canyou Zhang ${ }^{1}$, Chongguang Yang ${ }^{3}$, Yinyin Xia ${ }^{1}$, Jin Xing ${ }^{4}$, Guolong Zhang ${ }^{4}$, Lin X X ${ }^{5}$, Xiaomeng Wang ${ }^{6}$, Wei Lu', Jianwei Li ${ }^{8}$, Feiying Liu' ${ }^{9}$, Dingwen Lin ${ }^{9}$, Jianlin Wu ${ }^{10}$, Xin Shen ${ }^{11}$, Shuangyi Hou ${ }^{12}$, Yanling $\mathrm{Yu}^{13}$, Dongmei Hu${ }^{1}$, Chunyi Fu ${ }^{14}$, Lixia Wang ${ }^{1}$, Jun Cheng ${ }^{1 *}$ (I) and Hui Zhang ${ }^{1 *}$

\begin{abstract}
Background: Part of tuberculosis (TB) patients were missed if symptomatic screening was based on the main TB likely symptoms. This study conducted to compare the yield and relative costs of different TB screening algorithms in active case-finding in the whole population in China.

Methods: The study population was screened based on the TB likely symptoms through a face-to-face interview in selected 27 communities from 10 counties of 10 provinces in China. If the individuals had any of the enhanced TB likely symptoms, both chest X-ray and sputum tests were carried out for them furtherly. We used the McNemar test to analyze the difference in TB detection among four algorithms in active case-finding. Of four algorithms, two were from WHO recommendations including 1a/1c, one from China National Tuberculosis Program, and one from this study with the enhanced TB likely symptoms. Furthermore, a two-way ANOVA analysis was performed to analyze the cost difference in the performance of active case-finding adjusted by different demographic and health characteristics among different algorithms.

Results: Algorithm with the enhanced TB likely symptoms defined in this study could increase the yield of TB detection in active case-finding, compared with algorithms recommended by WHO ( $<<0.01$, Kappa 95\% Cl: 0. 93-0.99) and China NTP ( $p=0.03$, Kappa 95\% Cl: 0.96-1.00). There was a significant difference in the total costs among different three algorithms WHO $1 \mathrm{c} / 2 / 3(\mathrm{~F}=59.13, \mathrm{p}<0.01)$. No significant difference in the average costs for one active TB case screened and diagnosed through the process among Algorithms $1 \mathrm{c} / 2 / 3$ was evident $(F=2.78, p=0.07)$. The average costs for one bacteriological positive case through algorithm WHO 1a was about two times as much as the costs for one active TB case through algorithms WHO 1c/2/3.
\end{abstract}

Conclusions: Active case-finding based on the enhanced symptom screening is meaningful for TB case-finding and it could identify more active TB cases in time. The findings indicated that this enhanced screening approach cost more compared to algorithms recommend by WHO and China NTP, but the increased yield resulted in comparative

\footnotetext{
*Correspondence: chengjun@chinacdc.cn; zhanghui@chinacdc.cn

${ }^{1}$ National Center for Tuberculosis Control and Prevention, Chinese Center

for Disease Control and Prevention, Beijing, People's Republic of China

Full list of author information is available at the end of the article
}

(C) The Author(s) 2021. Open Access This article is licensed under a Creative Commons Attribution 4.0 International License, which permits use, sharing, adaptation, distribution and reproduction in any medium or format, as long as you give appropriate credit to the original author(s) and the source, provide a link to the Creative Commons licence, and indicate if changes were made. The images or other third party material in this article are included in the article's Creative Commons licence, unless indicated otherwise in a credit line to the material. If material is not included in the article's Creative Commons licence and your intended use is not permitted by statutory regulation or exceeds the permitted use, you will need to obtain permission directly from the copyright holder. To view a copy of this licence, visit http://creativecommons.org/licenses/by/4.0/. The Creative Commons Public Domain Dedication waiver (http://creativeco mmons.org/publicdomain/zero/1.0/) applies to the data made available in this article, unless otherwise stated in a credit line to the data. 
costs per patient. And it cost much more that only smear/bacteriological-positive TB cases are screened in active case-finding.

Keywords: Tuberculosis, Cost-effectiveness, Active case-finding, Screening

\section{Background}

Tuberculosis (TB) remains an infectious disease imposing severe hazards to human health. Moreover, TB listed as public health problems to urgently be addressed today together with HIV/AIDS and malaria by the World Health Organization (WHO) and was one of the major infectious diseases under key control in China [1]. Although China has made significant achievements in TB prevention and control owing to the long-term efforts by the government and all-level health sectors, the current situation is not optimistic [2]. China is still one of $30 \mathrm{~TB}$ high-burden countries in the world, and 886 thousand estimated incidenct TB patients in China ranks second in the world [3].

The combination of case-finding and curing TB patients is widely recognized as the most cost-effectiveness measure for TB prevention and control $[4,5]$. Due to limited resource, China currently adopts the strategy of passive case-finding and there are no more active case-finding measures except the symptom screening among the close contacts of pulmonary smear-positive TB patients and the individuals with HIV/AIDS [6]. The main TB likely symptoms include cough with expectoration $\geq 2$ weeks, hemoptysis and bloody sputum based on the national TB control program in China (China NTP) [7]. However, the national TB prevalence survey in 2010 showed that if symptomatic screening for TB was implemented based on the major suspected symptoms defined by China NTP, part of TB patients could be missed $[8,9]$.

Previous studies have reported that any cough and other symptoms such as chest pain and loss of weight, had particular low sensitivity and specificity for TB detection $[10,11]$. It meant that if the extended TB likely symptoms (such as chest pain) were applied as the criteria of further examinations for TB diagnosis, more cases would be detected. However, resource requirements for further tests may be prohibitive in some settings and a reason to opt for particular symptom screening [12].

Therefore, it was worthy to find a balance between the case detection and cost. We hypothesized that if more TB cases could be detected through the extended symptomatic screening compared with the general symptomatic screening strategies of WHO and China NTP. Here, we conduct this cross-section study in 10 provinces in China to compare the enhanced TB likely symptom screening algorithm in yield and cost of TB case finding with the screening algorithms from WHO and China NTP.

\section{Methods}

Ethics

The study protocol was approved by the Chinese center for disease control and prevention (China CDC) ethics committees (No. 201322). All participants before the enrollment signed the written informed consents. The written informed consent for the participants who were younger than 15 years old or patients with mental illness was obtained from their parent or guardian. All notified patients were referred to the local designated TB clinic or hospital for treatment according to national guidelines.

\section{Selection of study sites}

We applied a multi-stage sampling to create a representative sample from the Chinese mainland, with the following steps:

First, nine provinces of 31 provinces in terms of the east, central and west China respectively, and one of the four municipalities directly under the Central Government (Beijing, Shanghai, Tianjin, and Chongqing) were selected according to the willingness to participate. These were Jiangsu province, Zhejiang province and Guangdong province in eastern China, Henan province, Heilongjiang province and Hubei province in central China, Sichuan province, Guangxi Zhuang autonomous region and Yunnan province in western China, and Shanghai city.

Then, one county/district that had more than 500,000 people, was selected simple-randomly in each selected province.

Finally, the township/community was selected simple-randomly from each enrolled county. If the total number of the general population in selected township/community were less than 30,000 people, the neighborhood township/community would also be enrolled in the study site, till up to 30,000 people (Fig. 1) [13]. Totally ten townships and 17 communities were selected from 10 counties of 10 provinces/ municipalities.

\section{Study population}

The general population, who had been continuously living, working or studying in the survey sites for six months and above, including registered and 


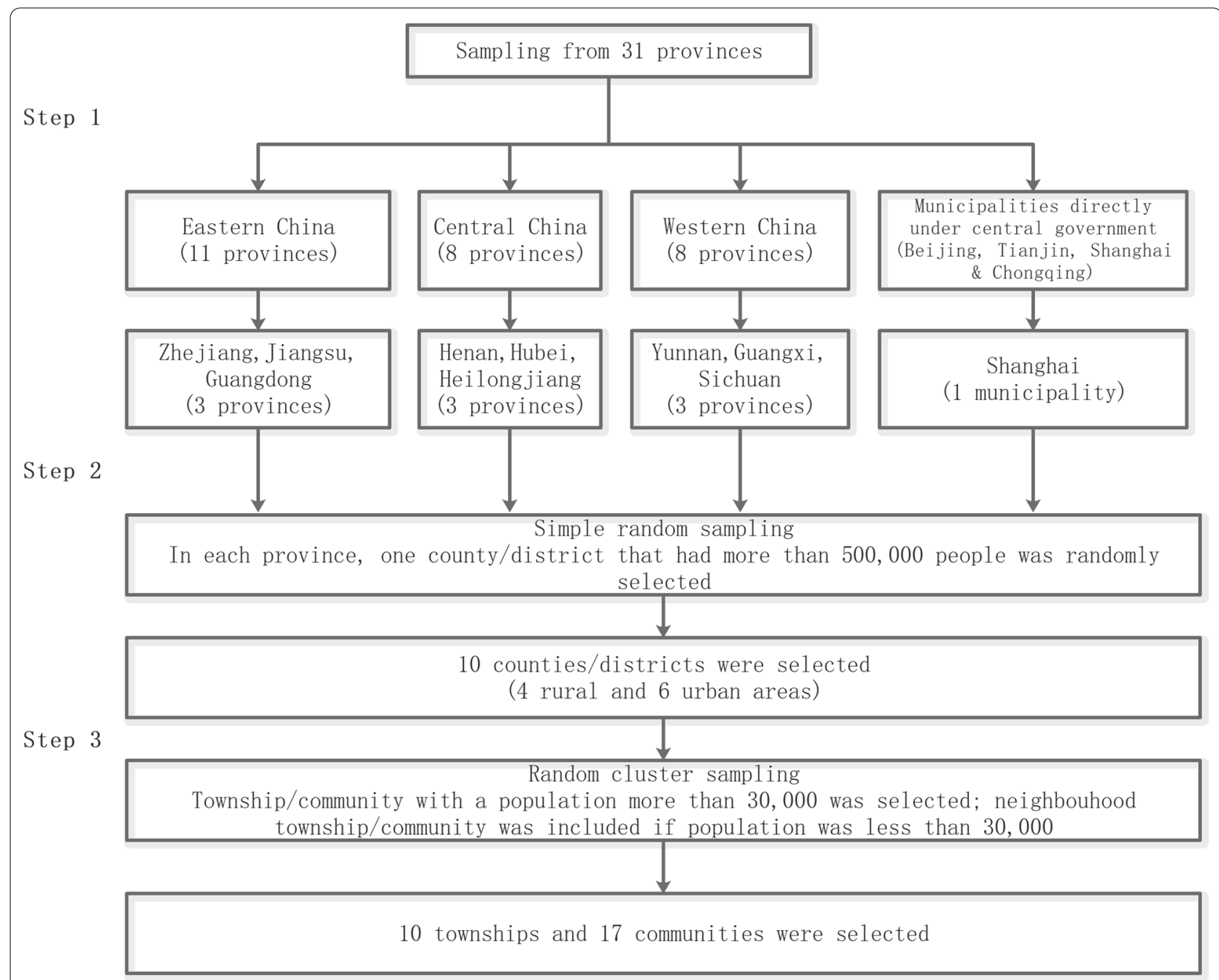

Fig. 1 The sampling procedure of this study

non-registered population, were enrolled in the study. Totally 320,590 of the general population were included in the selected townships/communities [13].

\section{Field investigation and diagnosis}

A face-to-face questionnaire-based inquiry was adopted to investigate whether the participant had any enhanced TB likely symptoms. The enhanced TB likely symptom was defined as any of the following conditions: 1) cough lasting longer than two weeks; 2) hemoptysis or bloody sputum; 3) cough longer than one week yet less than two weeks, and accompanied with any of the following symptoms: fever, night sweats, chest pain, loss of appetite, fatigue, or weight loss $(>3 \mathrm{~kg})$.

Participants with any of the enhanced TB likely symptoms were offered chest X-ray (CXR) examination and requested to submit three sputum samples (morning, night, and spot sputum) for both sputum smear microscopy and culture test.

If participants $<15$ years old had any enhanced TB likely symptoms, they were firstly supplied tuberculin skin test (PPD). Then, only those young participants with PPD induration $\geq 10 \mathrm{~mm}$ or blisters recieved CXR examination so that children and teenagers will not received unnecessary X-ray exposure.

Patients with smear-positive or culture-positive sputum were diagnosed as bacteriological positive TB. Patients with pulmonary tuberculosis (PTB) were defined as those with the bacteriological positive case and those diagnosed only by lesions on chest imaging known as clinically diagnosed cases.

The TB diagnosis group in each county composed of at least three health staffs, including a clinical doctor, a radiologist, and a laboratory technician, engaged in $\mathrm{TB}$ 
diagnosis. The criteria of TB diagnosis were compliance with the request of Diagnostic Criteria for Tuberculosis in China (WS288-2008) and the quality control was done according to the China National Guidelines [7].

\section{Data collection}

In our study, the participants were investigated by a specially trained investigation group each county for data collection based on the questionnaire from July to September 2013. The specially trained investigation group included researchers, health-care workers, community workers, and local government staff. The participants were interviewed for any enhanced TB likely symptoms. At the same time, their sex, age, ethnicity, occupation, marital status, educational level, medical history, smoking and drinking habit, socioeconomic status and TBrelated factors (Table 1) were collected as well. Moreover, their height and weight were measured to calculate BMI indicating their nutritional status.

All data on the questionnaire were entered in time and real-time double-checked by the online system developed especially for this study.

\section{TB screening algorithms with different paths}

To evaluate the yield of different symptom combination, four algorithms were compared, including Algorithm 1a/1c from WHO, Algorithm 2 from China National TB Control Program, and Algorithm 3 from our study.
Algorithm WHO 1a All people with a cough lasting longer than 2 weeks were investigated for TB. Sputum smear microscopy was considered as a second screening for people who have had a cough lasting longer than 2 weeks, and people with positive smear microscopy suggestive of TB should be diagnosed for TB. If sputum smear microscopy was negative and clinical suspicion was high, then considered further culture test for TB. The diagnosed TB cases were bacteriological positive cases. However, active TB cases who had negative sputum smear microscopy and the negative culture test could not be diagnosed due to a lack of chest X-ray.

Algorithm WHO 1c Further investigation for TB was done for persons with a cough lasting longer than 2 weeks. Chest radiography was considered as a second screening for people who screened positive when asked about symptoms, and people with an abnormal chest radiograph suggestive of TB should be evaluated by sputum smear microscopy and culture test for TB. Therefore, active TB cases who had negative sputum smear microscopy and negative culture test could be diagnosed due to the performance of symptom screening and Chest X-ray.

Algorithm 2 the screening algorithm based on China NTP: People with a cough lasting longer than 2 weeks, hemoptysis, or bloody sputum were investigated further for TB. Chest radiography was considered as a second

Table 1 Definitions of terms of data collocation

\begin{tabular}{|c|c|}
\hline Term & Definition \\
\hline Sex & Male, Female \\
\hline Age group & $0 \sim, 15 \sim, 25 \sim, 35 \sim, 45 \sim, 55 \sim, 65 \sim, 75 \sim, 85 \sim$ \\
\hline Ethnicity & Han, other \\
\hline Occupation & $\begin{array}{l}\text { Unknown, Child/Student, Housework/Unemployment; Teacher, Service provider, Health-care worker, } \\
\text { General worker, Farmer, Migrant worker, Retirement, Other }\end{array}$ \\
\hline Marital status & Married, Single/divorced, Unknown \\
\hline Education level & Unknown, Illiterate or semi-illiterate, Elementary school, Secondary school, College and above \\
\hline Previous TB case & Registered in TB Information Management System, and finished treatment or cure \\
\hline HIV/AIDS & $\begin{array}{l}\text { Registered in the local CDC database, who were diagnosed according to diagnostic criteria for HIV/AIDS } \\
\text { published by the National Health Commission of the People's Republic of China in } 2008\end{array}$ \\
\hline Known diabetes & $\begin{array}{l}\text { Recorded on the Citizen Health Management Files as diagnosed with Diabetes (fasting plasma glucose } \\
\text { level } \geq 7.0 \mathrm{mmol} / \mathrm{L} \text {, or } 2 \text {-h plasma glucose level } \geq 11.1 \mathrm{mmol} / \mathrm{L} \text { ), plus those using medicine to control } \\
\text { blood glucose by self-report }\end{array}$ \\
\hline Close contact & Living with a new active PTB case for at least 7 days in the 3 months before diagnosis \\
\hline BMI level [14] & 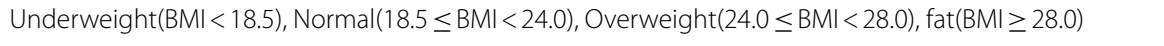 \\
\hline Tobacco use & Ever smoked tobacco by self-report \\
\hline Drinking habit & Drinking more than one unit(21 g pure alcohol) per week by self-report \\
\hline Chronic bronchitis & Chronic bronchitis history by self-report \\
\hline Average family annual income per capita & $\begin{array}{l}\text { Average annual income per capita of urban family = RMB 27,000 (USD 3970), average annual income per } \\
\text { capita of rural family= RMB } 8000 \text { (USD 1176), (USD 1=RMB 6.8) }\end{array}$ \\
\hline Average family living area per capita & Average of urban family $=29 \mathrm{~m}^{2}$, an average of rural family $=31 \mathrm{~m}^{2}$ \\
\hline
\end{tabular}


screening where chest X-ray is available for people who screened positive when asked about symptoms. People with suspected symptoms or an abnormal chest radiograph suggestive of TB should be evaluated by sputum smear microscopy and culture for TB. So active TB case with negative sputum smear microscopy and negative culture test could be diagnosed as well, beside bacteriological positive TB case.

Algorithm 3 (The enhanced symptoms screening algorithm for TB): Persons with any of the following 3 options suggestive of TB should be further evaluated for TB. (1) cough lasting longer than 2 weeks; (2) hemoptysis or bloody sputum; (3) cough longer than 1 week yet less than 2 weeks, and accompanied with any of the following symptoms: fever, night sweats, chest pain, loss of appetite, fatigue, or weight $\operatorname{loss}(>3 \mathrm{~kg})$. Chest radiography was considered as a second screening for people who screened positive when asked about symptoms. People with suspected symptoms or an abnormal chest radiograph suggestive of TB should be evaluated by sputum smear microscopy and culture for TB. The TB diagnostic process was the same as Algorithm 2.

Algorithms WHO 1a/1c for screening and diagnosis were one of the recommendations from WHO [1517]. Of 3 options of Algorithm 3, option (3) had been enhanced added to the definition of the TB likely symptoms in this study compared with Algorithm 2 the definition from China NTP. (Table 2) The participants had received all screening procedures in our study according to Algorithm 3, so the study population and case detection of algorithm WHO $1 \mathrm{a} / 1 \mathrm{c}$ and 2 were extracted according to the definition of different algorithms.

\section{Costs of active case screening}

The cost was calculated among different screening algorithms based on the standard criteria of each component of the screening process, thereof 0.15 dollars per persontime for primary household screening by village health workers, 9.0 dollars per time for Chest X-ray, 3.9 dollars per time for sputum smear, 4.8 dollars per time for sputum culture[13]. Algorithm WHO 1a needed $\$ 8.85$ for each person with suspected symptoms, and algorithm WHO 1c/2/3 needed $\$ 17.85$. The functions of total cost of different algorithms were as followed:

Function 1:

$$
\begin{aligned}
& \text { Totalcostofscreening }(\text { AlgorithmWHO1a) } \\
& =\text { numberofstudypopulation } \times \$ 0.15 \\
& \quad+\text { numberofparticipatnswithsuspectedsymptom } \\
& \quad \times(\$ 3.9+\$ 4.8)
\end{aligned}
$$

Function 2:

$$
\begin{aligned}
& \text { Totalcostofscreening }(\text { AlgorithmWHO1c/2/3) } \\
& \begin{aligned}
= & \text { numberofstudypopulation } \times \$ 0.15 \\
& + \text { numberofparticipatnswithsuspectedsymptom } \\
& \times(\$ 9+\$ 3.9+\$ 4.8)
\end{aligned}
\end{aligned}
$$

\section{Statistical analysis}

The prevalence of TB likely symptoms and TB cases was calculated. The McNemar test was used to analyze the difference in case detection between every two algorithms. Two-way ANOVA test was performed to evaluate the difference in the costs of different algorithms adjusted by demographic and health characteristics. Two-sided $p<0.05$ was considered to be significant. All tests were performed using SAS 9.4 (SAS Institute, Cary, NC, USA).

\section{Results}

\section{Demographic and health characteristics}

There was a total of 320,590 eligible population, of which

\begin{tabular}{|c|c|c|c|c|c|c|}
\hline \multirow[t]{2}{*}{ Algorithm } & \multicolumn{3}{|c|}{ Household primary screening } & \multirow[t]{2}{*}{ Chest X-ray } & \multirow[t]{2}{*}{ Smear } & \multirow[t]{2}{*}{ Culture } \\
\hline & 1) & 2) & 3) & & & \\
\hline WHO 1a & $x$ & & & & $x$ & $x$ \\
\hline WHO 1c & $x$ & & & $x$ & $x$ & $x$ \\
\hline 2 & $x$ & $x$ & & $x$ & $x$ & $x$ \\
\hline 3 & $x$ & $x$ & $x$ & $x$ & $x$ & $x$ \\
\hline
\end{tabular}
299,610 persons $(93.5 \%)$ were enrolled in the survey. Inaddtion to the questionnaire investigation, the CXR examination were performed for the people with the enhanced suspected symptoms. Males accounted for $50.2 \%(150,461)$, and females for $49.8 \%(149,149)$. The mean age was 39-year-old (median 40-year-old) Table 3.

Table 2 The screening procedure through algorithm WHO 1a/1c, 2, and 3

(1) cough lasting longer than two weeks; (2) hemoptysis or bloody sputum; (3) cough longer than one week yet less than two weeks, and accompanied with any of the following symptoms: fever, night sweats, chest pain, loss of appetite, fatigue, or weight loss $(>3 \mathrm{~kg})$ 


\section{Comparison in case detection between each two algorithms}

There were significant differences in the case detection of active TB cases in the comparisons between Algorithm WHO 1c and 3 (p<0.01, Kappa 95\%CI: 0. 93-0.99) and between Algorithms 2 and 3 ( $\mathrm{p}=0.03$, Kappa 95\% CI: 0.96-1.00). No significant difference was observed between any other two algorithms in the same type of TB detection. (Table 4).

\section{The yield of different algorithms and their costs}

Table 5 summarized the numbers of people screened in this study via different algorithms, the corresponding numbers of people with suspected symptoms, active tuberculosis cases diagnosed, and the related costs.

In this study, both Algorithm $1 \mathrm{c}$ and Algorithm 2 notified one active TB case per average 22 (2328/105, 2407/108) persons with TB likely symptoms screened. Compared with algorithm WHO 1c, Algorithm 3 identified 8 (113-105) more active TB cases among an additional 348 (2676-2328) persons screened with the option 3) (cough longer than 1 week yet less than 2 weeks, and accompanied with any of the following symptoms: fever, night sweats, chest pain, loss of appetite, fatigue, or weight loss $(>3 \mathrm{~kg}))$, which indicated an additional average of $44(348 / 8)$ persons to be screened to diagnose one more active TB case. Meanwhile, Algorithm 3 required an additionally screening of 269 (2676-2407) persons with the option 3) compared to the Algorithm 2 for the diagnosis of 5 (113-108) more active TB cases. It suggested that an average of $54(269 / 5)$ persons with option 3 ) of the enhanced TB likely symptoms screening beyond the TB likely symptoms defined by Algorithm 2 needed to be screened further to detect one more active TB case through Algorithm 3. When Algorithm 2 based on current China NTP was considered as "Golden standard", 1) through Algorithm 3, the false positive rate was $1.7 / 100,000$ and false negative rate was $0 ; 2$ ) through Algorithm WHO 1c, the false positive rate 0 and false negative rate was 2.8\%. Because Algorithm WHO 1a did not conduct CXR test as one of screening procedure, so it was not reasonable to compare between Algorithm 2 and WHO $1 \mathrm{~A}$.

Of 299,610 persons, 2328 with the TB likely symptoms defined by Algorithm WHO 1a/1c, were identified. Therefore, 37 bacteriological-positive TB cases via algorithm WHO 1a were diagnosed and the total costs of the processes of Algorithm WHO 1a were $\$ 65,195.1$, which meant it cost $\$ 1762.0$ per bacteriological-positive TB case diagnosed by Algorithm WHO 1a. There were as many as the persons with the TB likely symptoms identified and 105 active TB cases diagnosed via algorithm WHO 1c. The total costs of Algorithm WHO 1c were
Table 3 Demographic characteristics of the enrolled population in China in 2013

\begin{tabular}{|c|c|c|}
\hline Variable & Number & Proportion(\%) \\
\hline All & 299,610 & 100.00 \\
\hline \multicolumn{3}{|l|}{ Sex } \\
\hline Male & 150,461 & 50.2 \\
\hline Female & 149,149 & 49.8 \\
\hline \multicolumn{3}{|l|}{ Age group } \\
\hline $0 \sim$ & 51,537 & 17.2 \\
\hline $15 \sim$ & 32,312 & 10.8 \\
\hline $25 \sim$ & 40,259 & 13.4 \\
\hline $35 \sim$ & 48,363 & 16.1 \\
\hline $45 \sim$ & 47,675 & 15.9 \\
\hline $55 \sim$ & 45,195 & 15.1 \\
\hline $65 \sim$ & 21,685 & 7.2 \\
\hline $75 \sim$ & 10,658 & 3.6 \\
\hline $85 \sim$ & 19,26 & 0.6 \\
\hline \multicolumn{3}{|l|}{ Ethnicity } \\
\hline Han & 268,145 & 89.5 \\
\hline Other & 31,465 & 10.5 \\
\hline \multicolumn{3}{|l|}{ Occupation } \\
\hline Unknown & 13,757 & 4.6 \\
\hline Child/Student & 63,867 & 21.3 \\
\hline Housework/Unemployment & 17,627 & 5.9 \\
\hline Teacher & 3103 & 1.0 \\
\hline Service provider & 6132 & 2.1 \\
\hline Health-care worker & 1155 & 0.4 \\
\hline General worker & 37,639 & 12.6 \\
\hline Farmer & 104,926 & 35.0 \\
\hline Migrant worker & 8951 & 3.0 \\
\hline Retirement & 19,999 & 6.7 \\
\hline Other & 22,454 & 7.5 \\
\hline \multicolumn{3}{|l|}{ Marital status } \\
\hline Married & 190,635 & 63.6 \\
\hline Single/divorced & 99,173 & 33.1 \\
\hline Unknown & 9802 & 3.3 \\
\hline \multicolumn{3}{|l|}{ Education level } \\
\hline Unknown, & 10,491 & 3.5 \\
\hline Illiterate or semi-illiterate, & 37,016 & 12.4 \\
\hline Elementary school, & 77,294 & 25.8 \\
\hline Secondary school, & 146,082 & 48.8 \\
\hline College and above & 28,727 & 9.6 \\
\hline \multicolumn{3}{|l|}{ Previous TB case } \\
\hline Yes & 1595 & 0.5 \\
\hline No & 298,015 & 99.5 \\
\hline \multicolumn{3}{|l|}{ HIV/AIDS } \\
\hline Yes & 59 & $<0.1$ \\
\hline No & 299,551 & $>99.9$ \\
\hline \multicolumn{3}{|l|}{ Known diabetes } \\
\hline Yes & 5150 & 1.7 \\
\hline No & 294,460 & 98.3 \\
\hline
\end{tabular}


Table 3 (continued)

\begin{tabular}{|c|c|c|}
\hline Variable & Number & Proportion(\%) \\
\hline \multicolumn{3}{|l|}{ Close contact } \\
\hline Yes & 378 & 0.1 \\
\hline No & 299,232 & 99.9 \\
\hline \multicolumn{3}{|l|}{ BMI level } \\
\hline$<18.5$ & 18,031 & 6.0 \\
\hline $18.5 \leq \mathrm{BMI}<24.0$ & 160,854 & 53.7 \\
\hline $24.0 \leq \mathrm{BMl}<28.0$ & 57,858 & 19.3 \\
\hline $\mathrm{BMI} \geq 28.0$ & 11,457 & 3.8 \\
\hline Unknown & 51,410 & 17.2 \\
\hline \multicolumn{3}{|l|}{ Tobacco use } \\
\hline Never smoke & 196,412 & 65.6 \\
\hline Prior smoker & 4948 & 1.7 \\
\hline Current smoker & 46,854 & 15.6 \\
\hline Unknown & 51,396 & 17.2 \\
\hline \multicolumn{3}{|l|}{ Drinking habit } \\
\hline Never drink & 197,481 & 65.9 \\
\hline Prior alcohol user & 5,107 & 1.7 \\
\hline Current alcohol user & 45,348 & 15.1 \\
\hline Unknown & 51,674 & 17.3 \\
\hline \multicolumn{3}{|l|}{ Chronic bronchitis } \\
\hline Yes & 3381 & 1.1 \\
\hline No & 244,041 & 81.5 \\
\hline Unknown & 52,188 & 17.42 \\
\hline \multicolumn{3}{|l|}{ Pneumoconiosis } \\
\hline Yes & 178 & $<0.1$ \\
\hline No & 247,993 & 82.8 \\
\hline Unknown & 51,439 & 17.2 \\
\hline \multicolumn{3}{|c|}{ Average family annual income per capita* } \\
\hline Higher than average & 91,203 & 33.0 \\
\hline Lower than average & 185,220 & 67.0 \\
\hline \multicolumn{3}{|c|}{ Average family living area per capita* } \\
\hline Higher than average & 105,812 & 38.2 \\
\hline Lower than average & 171,007 & 61.8 \\
\hline
\end{tabular}

Note: * Missing values existed
$\$ 86,147.1$, which indicated that it cost $\$ 820.4$ per active TB case diagnosed by algorithm WHO 1c. Additionally, algorithm 2 cost $\$ 810.6(\$ 87,545.4 / 108)$ for one active TB case, among 108 active TB cases from 2407 persons with suspected symptoms. Finally, the cost of $\$ 816.9$ $(\$ 92,306.7 / 113)$ for one active TB case was estimated among 113 active TB cases from 2676 persons with suspected symptoms defined by algorithm 3 .

A two-way ANOVA adjusted by different TB types and screening algorithms revealed that there was a significant difference in the total costs of screening for active TB cases in different Algorithms WHO $1 \mathrm{c} / 2 / 3(\mathrm{~F}=59.13, \mathrm{p}<0.01$, Additional file 1: Appendix S1). Both the number of TB cases and the total costs through algorithm 3 were maximum, compared with Algorithms 1c/2. However, no significant difference was evident in the average costs for one active $\mathrm{TB}$ case screened and diagnosed through the process among Algorithms WHO 1c/2/3 $(\mathrm{F}=2.78, \mathrm{p}=0.07$, Additional file 1: Appendix S1). On the other hand, the average costs for one bacteriological positive case through Algorithm WHO 1a was about two times as much as the costs for one active TB case through Algorithms WHO 1c/2/3 (Fig. 2).

\section{Discussion}

Compared with WHO recommendation and China NTP guideline, more persons with TB likely symptoms were identified through Algorithm 3, and the total costs of this algorithm increased significantly as well. Furthermore, the introduction of the additional enhanced TB likely symptoms induced more active TB cases diagnosed. However, the average costs for one active TB case diagnosis through Algorithm 3 were no signs of a difference compared with Algorithms WHO 1c and 2. While much more costs should spend if the screening targeted at a bacteriological positive TB case.

Table 4 McNemar's test for detection rate between different symptom screening algorithms, p-value (95\%Cl for Kappa)

\begin{tabular}{|c|c|c|c|c|c|c|c|}
\hline \multirow[t]{3}{*}{ Algorithm } & \multirow[t]{3}{*}{ Type of patient } & \multicolumn{6}{|c|}{ Type of patient in different algorithms } \\
\hline & & 2 & 2 & 2 & 3 & 3 & 3 \\
\hline & & Smear + & Bact + & Active TB & Smear + & Bact + & Active TB \\
\hline WHO 1a & Smear+ & $0.32(0.95,1.00)$ & - & - & $0.05(0.86,1.00)$ & - & - \\
\hline WHO 1a & Bact + & - & $0.32(0.96,1.00)$ & - & - & $0.05(0.90,1.00)$ & - \\
\hline WHO 1c & Smear + & $0.32(0.95,1.00)$ & - & - & $0.05(0.86,1.00)$ & - & - \\
\hline WHO 1c & Bact + & - & $0.32(0.96,1.00)$ & - & - & $0.05(0.90,1.00)$ & - \\
\hline WHO 1c & Active TB & - & - & $0.08(0.97,1.00)$ & - & - & $<0.01(0.93,0.99)$ \\
\hline 2 & Smear + & - & - & - & $0.08(0.89,1,00)$ & - & - \\
\hline 2 & Bact + & - & - & - & - & $0.08(0.92,1.00)$ & - \\
\hline 2 & Active TB & - & - & - & - & - & $0.03(0.96,1.00)$ \\
\hline
\end{tabular}


Table 5 Yield and relative costs of different symptom screening algorithms

\begin{tabular}{|c|c|c|c|c|c|c|c|}
\hline \multirow[t]{2}{*}{ Type of patient } & \multirow[t]{2}{*}{ Algorithm } & \multirow[t]{2}{*}{ Symptom No } & \multirow[t]{2}{*}{ No. of TB case } & \multicolumn{2}{|c|}{ TB detection and screening } & \multicolumn{2}{|c|}{ Relative costs } \\
\hline & & & & $\begin{array}{l}\text { Detection } \\
\text { proportion*(\%) }\end{array}$ & $\begin{array}{l}\text { Screening No. } \\
\text { per case }\end{array}$ & Total costs & $\begin{array}{l}\text { Average costs } \\
\text { for one case }\end{array}$ \\
\hline \multirow[t]{4}{*}{ Smear + } & $1 \mathrm{a}$ & 2328 & 27 & 1.16 & 11,097 & $65,195.1$ & 2414.6 \\
\hline & $1 c$ & 2328 & 27 & 1.16 & 11,097 & $86,147.1$ & 3190.6 \\
\hline & 2 & 2407 & 28 & 1.16 & 10,700 & $87,545.4$ & 3126.6 \\
\hline & 3 & 2676 & 31 & 1.16 & 9665 & $92,306.7$ & 2977.6 \\
\hline \multirow[t]{4}{*}{ Bact +} & $1 a$ & 2328 & 37 & 1.59 & 8098 & $65,195.1$ & 1762.0 \\
\hline & $1 \mathrm{c}$ & 2328 & 37 & 1.59 & 8098 & $86,147.1$ & 2328.3 \\
\hline & 2 & 2407 & 38 & 1.58 & 7884 & $87,545.4$ & 2303.8 \\
\hline & 3 & 2676 & 41 & 1.53 & 7308 & $92,306.7$ & 2251.4 \\
\hline \multirow[t]{3}{*}{ Active TB } & $1 c$ & 2328 & 105 & 4.51 & 2853 & $86,147.1$ & 820.4 \\
\hline & 2 & 2407 & 108 & 4.49 & 2774 & $87,545.4$ & 810.6 \\
\hline & 3 & 2676 & 113 & 4.22 & 2651 & $92,306.7$ & 816.9 \\
\hline
\end{tabular}

*Detection proportion among people with suspected symptoms

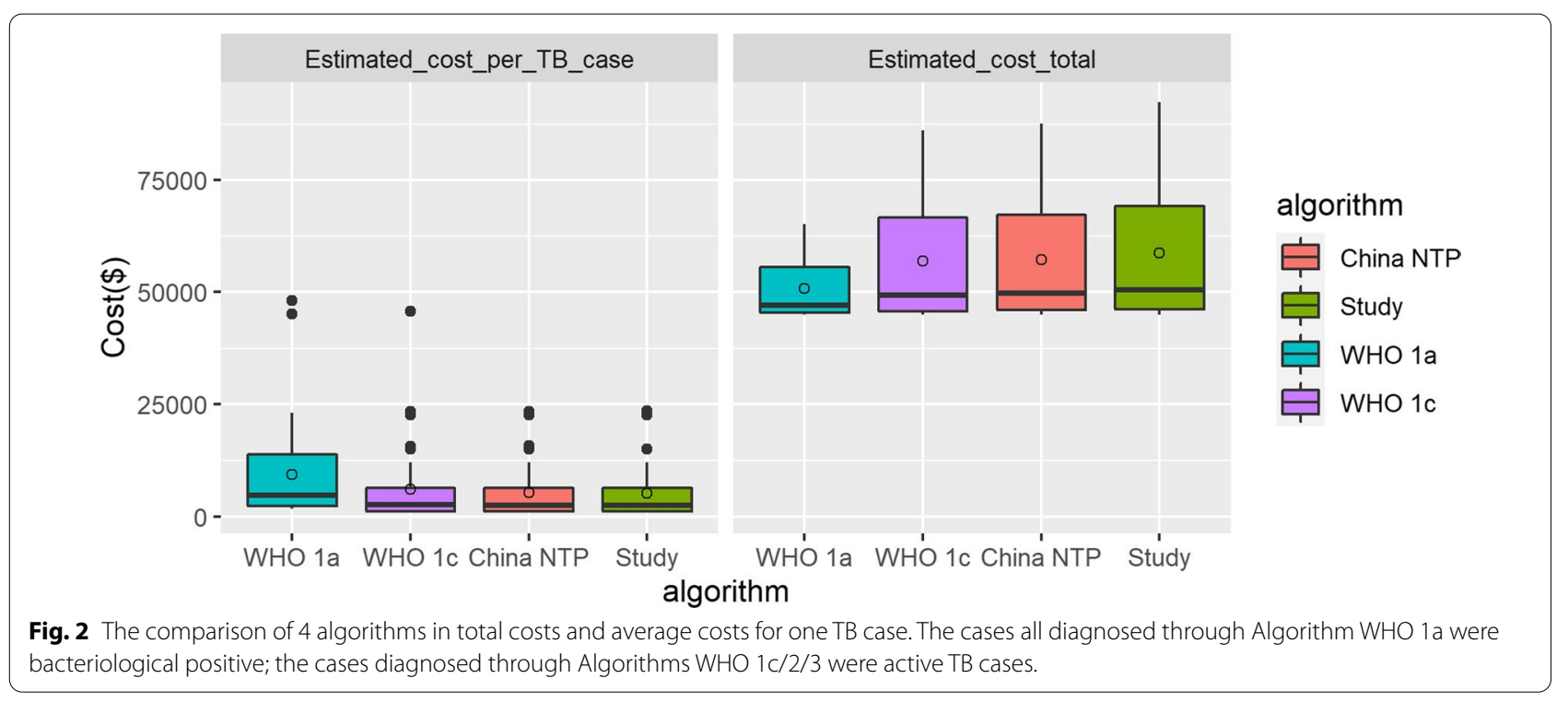

During the past few years, there has been an intensified discussion about using active case-finding, or screening, as a possible complement to the predominant approach of "passive case-finding". The primary objective of screening is to ensure that active TB is detected early to reduce the risk of poor disease outcomes and the adverse social and economic consequences of the disease, as well as help reduce TB transmission [16]. Although when a country is striving to eliminate $\mathrm{TB}$ and needs to invest additional resources to effectively reach those who are hardest to reach, active screening may be a crucial part of the response to TB [18]. Besides, the alternative screening algorithms and their costs should be considered based on sufficient evidence as well, especially in recourse-constrained high-burden countries [19]. In our study, the additional enhanced suspected symptoms during the screening could identified more active TB. And this algorithm in the costs for one active TB case was comparable to the algorithms of WHO/China NTP. Thus, it will be of interest to take active TB screening potentially in the low TB burden countries with sufficient resources or the resource-constrained countries striving to eliminate TB. The enhanced suspected symptoms for active TB screening was an alternative and could identify more active TB cases to reduce TB transmission. However, that was undoubtedly low cost-effectiveness for only 
screening smear/bacteriological positive TB cases in the active case-finding or screening. Our study indicated that the average costs for detecting one bacteriological positive case through Algorithm WHO 1a were approximately double as the costs for detecting one active $\mathrm{TB}$ case through Algorithms WHO 1c/2/3.

The costs of active case-finding appears to be different in a variety of settings [20]. The costs of active case-finding were more than passive case-finding [21]. However, the enhanced TB suspected symptoms in our study could help diagnose more active TB cases sooner, which was similar to that found in comparable studies [22]. Furthermore, compared with other studies in active screening for $\mathrm{TB}$, our average screening costs for one active TB case was less than the same study in Russian Federation, despite the same level of TB incidence in both countries, but more than the studies in the higher TB-incidence countries [3, 21, 23, 24]. In addition, if the active case-finding might be performed in targeted high-risk groups, the costs of performance could decrease undoubtedly.

Our study is population-based research. These findings provided insights into the yield and costs of different active case-finding approaches. Our research groups had tried the best to keep the quality control. Although the large sample size allowed further analysis in different demographic and health characteristics, some inevitable missing values, like other extensive population studies, made these findings accompany with uncontrolled little information bias. And there might be false positives including particularly as $64 \%$ of the diagnoses through Algorithm 3 were not confirmed by smear or culture.

\section{Conclusions}

Active case-finding based on the enhanced symptom screening is meaningful for TB case-finding and it could identify more active TB cases in time. The findings indicated that this active case-finding cost more into enhanced screening but the increased yield of active TB cases resulted in comparative costs per patient, despite increased total costs. This active case-finding potentially in the low TB burden countries with sufficient resources or the resource-constrained countries striving to eliminate $\mathrm{TB}$ as an alternative could identify more active TB cases to reduce TB transmission. And it cost much more that only smear/bacteriological positive $\mathrm{TB}$ cases are screened in active case-finding.

\section{Abbreviations}

ACF: Active case finding; AIDS: Acquired immune deficiency syndrome; BMI: Body mass index; Cl: Confidence interval; CXR: Chest X-ray; HIV: Human immunodeficiency virus; PTB: Pulmonary tuberculosis; TB: Tuberculosis.

\section{Supplementary Information}

The online version contains supplementary material available at https://doi. org/10.1186/s12879-021-06486-w.

Additional file 1. Results of Symptom screening and TB detection in different algorithms and variables..

\section{Acknowledgements}

We would like to thank all the health care workers and staff for their hard work in research sites, including the provincial CDCs, the local CDCs, and the primary health centers/institutes. The study sites were located in Jiangsu Province, Zhejiang Province, Guangdong Province and Shanghai of eastern China, Henan Province, Heilongjiang Province and Hubei Province of central China, and Sichuan Province, Guangxi Zhuang Autonomous Region and Yunnan Province of western China.

\section{Authors' contributions}

Substantial contributions to the conception and design of the work and the analyses and interpretation of data for the work: LXW, GXH, HZ, JC, FZ, CYZ, YYX, DMH; Data collection and performed the experiments: JX, GLZ, LX, XMW, $W L$, JWL, FYL, DWL, JLW, XS, SYH, YLY; Drafting the work or revising it critically for important intellectual content: FZ, CYZ, CGY, CYF; Final approval of the version to be published: $H Z, J C, L X W$. All authors read and finally approved the manuscript draft for publication. All authors read and approved the final manuscript.

\section{Funding}

Funding for this study was obtained by the National Twelfth and Thirteenth Five-year Major-Scientific Projects of Infectious Diseases in China (Grant Number: 2013ZX10003-004-001, 2017ZX10201-302-001) from the Ministry of science and technology of China. These projects are independent funding schemes of the Ministry of science and technology of China.

\section{Availability of data and materials}

The National Center for Tuberculosis Control and Prevention (NCTB) is the custodian of the data for this study. The data are not accessible online but may be made available upon written request to the NCTB through the authors, if in line with the Ethical Review Board guidelines.

\section{Declarations}

Ethics approval and consent to participate

The protocol was approved by China CDC Ethical Review Committee in the Chinese Center for Disease Control and Prevention (No. 201322). The participants older than 18 years provided written informed consent by themselves and written informed consent was obtained from parents/guarantees for the participants under 18-year-old.

\section{Consent for publication}

Not applicable.

\section{Competing interests}

None of the authors have expressed any conflict of interest.

\section{Author details}

${ }^{1}$ National Center for Tuberculosis Control and Prevention, Chinese Center for Disease Control and Prevention, Beijing, People's Republic of China. ${ }^{2} \mathrm{Clini}-$ cal Trial Center, Beijing Hospital, National Center of Gerontology, Institute of Geriatric Medicine, Chinese Academy of Medical Sciences, Beijing, People's Republic of China. ${ }^{3}$ Department of Epidemiology of Microbial Diseases, Yale School of Public Health, New Haven, CT, USA. ${ }^{4}$ Institute of Tuberculosis Control and Prevention, Henan Provincial Center for Disease Control and Prevention, Zhengzhou, Henan, People's Republic of China. ${ }^{5}$ Division of Tuberculosis Control and Prevention, Yunnan Provincial Center for Disease Control and Prevention, Kunming, Yunnan, People's Republic of China. ${ }^{6}$ Institute of TB Control, Zhejiang Provincial Center for Disease Control and Prevention, Hangzhou,

Zhejiang, People's Republic of China. ${ }^{7}$ Department of Chronic Communicable 
Disease, Jiangsu Provincial Center for Disease Control and Prevention, Nanjing, Jiangsu, People's Republic of China. ${ }^{8}$ Center for Tuberculosis Control of Guangdong Province, Guangzhou, Guangdong, People's Republic of China. ${ }^{9}$ Guangxi Provincial Center for Disease Control and Prevention, Nanning, Guangxi Zhuang Autonomous Region, People's Republic of China. ${ }^{10}$ Sichuan Provincial Center for Disease Control and Prevention, Chengdu, Sichuan, People's Republic of China. ${ }^{11}$ Shanghai Municipal Center for Disease Control and Prevention, Shanghai, People's Republic of China. ${ }^{12}$ Hubei Provincial Center for Disease Control and Prevention, Wuhan, Hubei, People's Republic of China. ${ }^{13}$ Heilongjiang Provincial Center for Tuberculosis Control and Prevention, Harbin, Heilongjiang, People's Republic of China. ${ }^{14}$ Department of Emergency Medicine, Beijing Hospital, National Center of Gerontology, Institute of Geriatric Medicine, Chinese Academy of Medical Sciences, Beijing, People's Republic of China.

Received: 14 April 2020 Accepted: 28 July 2021

Published online: 13 August 2021

\section{References}

1. Xu CH, Jeyashree K, Shewade HD, Xia YY, Wang LX, Liu Y, Zhang H, Wang L. Inequity in catastrophic costs among tuberculosis-affected households in China. Infect Dis Poverty. 2019;8(1):46.

2. Wang L, Zhang H, Ruan Y, Chin DP, Xia Y, Cheng S, Chen M, Zhao Y, Jiang S, Du X, et al. Tuberculosis prevalence in China, 1990-2010: a longitudinal analysis of national survey data. Lancet. 2014;383(9934):2057-64.

3. World Health Organization. Global tuberculosis report, 2019. Switzerland: World Health Organization; 2019.

4. Wang L, Liu J, Chin DP. Progress in tuberculosis control and the evolving public-health system in China. Lancet. 2007;369(9562):691-6.

5. Chen JO, Qiu YB, Rueda ZV, Hou JL, Lu KY, Chen LP, Su WW, Huang L, Zhao $\mathrm{F}$, Li T, et al. Role of community-based active case finding in screening tuberculosis in Yunnan province of China. Infect Dis Poverty. 2019;8(1):92.

6. Liu E, Cheng S, Wang X, Hu D, Zhang T, Chu C. A systematic review of the investigation and management of close contacts of tuberculosis in China. J Public Health (Oxf). 2010;32(4):461-6.

7. $\mathrm{MOH}, \mathrm{CDCC}$. Guidelines for implementing the national tuberculosis control program in China. Beijing: Pecking Union Medical College Press; 2009.

8. Wang Y. Data compilation of the fifth national tuberculosis epidemiological sampling survey. Beijing: Military Medical Science Press; 2011.

9. Cheng J, Wang L, Zhang H, Xia Y. Diagnostic value of symptom screening for pulmonary tuberculosis in China. PLoS ONE. 2015;10(5):e0127725.

10. van't Hoog A, Langendam M, Mitchell E, Cobelens F, Sinclair D, Leeflang M. A systematic review of the sensitivity and specificity of symptom-and chest-radiography screening for active pulmonary tuberculosis in HIVnegative persons and persons with unknown HIV status. REPORT-Version March 2013. Geneva: World Health Organization; 2013.

11. van't Hoog AH. Meme HK, Laserson KF, Agaya JA, Muchiri BG, Githui WA, Odeny LO, Marston BJ, Borgdorff MW: Screening strategies for tuberculosis prevalence surveys: the value of chest radiography and symptoms. PLoS ONE. 2012;7(7):e38691.
12. Van't Hoog AH, Onozaki I, Lonnroth K. Choosing algorithms for TB screening: a modelling study to compare yield, predictive value and diagnostic burden. BMC Infect Dis. 2014;14:532.

13. Zhang C, Ruan Y, Cheng J, Zhao F, Xia Y, Zhang H, Wilkinson E, Das M, Li J, Chen W, et al. Comparing yield and relative costs of WHO TB screening algorithms in selected risk groups among people aged 65 years and over in China, 2013. PLoS ONE. 2017;12(6):e0176581.

14. Criteria of weight for adults. Beijing: National Health and Family Planning Commission of the People's Republic of China: 2013.

15. World Health Organization. Systematic screening for active tuberculosis: an operational guide. Geneva: World Health Organization; 2015.

16. World Health Organization. WHO guidelines approved by the guidelines review committee. Systematic screening for active tuberculosis: principles and recommendations. Geneva: World Health Organization; 2013.

17. van't Hoog A, Langendam M, Mitchell E, Cobelens F, Sinclair D, Leeflang $\mathrm{M}$, Lonnroth K. A systematic review of the sensitivity and specificity of symptom-and chest-radiography screening for active pulmonary tuberculosis in HIV-negative persons and persons with unknown HIV status REPORT-Version March 2013. Geneva: World Health Organization; 2013.

18. Broekmans JF, Migliori GB, Rieder HL, Lees J, Ruutu P, Loddenkemper R, Raviglione MC, World Health Organization IUAT, Lung D, Royal Netherlands Tuberculosis Association Working G. European framework for tuberculosis control and elimination in countries with a low incidence Recommendations of the World Health Organization (WHO), International Union Against Tuberculosis and Lung Disease (IUATLD) and Royal Netherlands Tuberculosis Association (KNCV) Working Group. Eur Respir J. 2002;19(4):765-75.

19. Martinez L, Shen Y, Handel A, Chakraburty S, Stein CM, Malone LL, Boom WH, Quinn FD, Joloba ML, Whalen CC, et al. Effectiveness of WHO's pragmatic screening algorithm for child contacts of tuberculosis cases in resource-constrained settings: a prospective cohort study in Uganda. Lancet Respir Med. 2018;6(4):276-86.

20. Ho J, Fox GJ, Marais BJ. Passive case finding for tuberculosis is not enough. Int J Mycobacteriol. 2014;5(4):374-8.

21. Bogdanova E, Mariandyshev O, Hinderaker SG, Nikishova E, Kulizhskaya A, Sveshnikova O, Grjibovski A, Heldal E, Mariandyshev A. Mass screening for active case finding of pulmonary tuberculosis in the Russian Federation: how to save costs. Int JTuberc Lung Dis. 2019;23(7):830-7.

22. Field SK, Escalante P, Fisher DA, Ireland B, Irwin RS. Cough due to TB and other chronic infections: CHEST guideline and expert panel report. Chest. 2018;153(2):467-97.

23. Hussain H, Mori AT, Khan AJ, Khowaja S, Creswell J, Tylleskar T, Robberstad B. The cost-effectiveness of incentive-based active case finding for tuberculosis (TB) control in the private sector Karachi, Pakistan. BMC Health Serv Res. 2019;19(1):690.

24. Machekera SM, Wilkinson E, Hinderaker SG, Mabhala M, Zishiri C, Ncube RT, Timire C, Takarinda KC, Sengai T, Sandy C. A comparison of the yield and relative cost of active tuberculosis case-finding algorithms in Zimbabwe. Public health action. 2019;9(2):63-8.

\section{Publisher's Note}

Springer Nature remains neutral with regard to jurisdictional claims in published maps and institutional affiliations.
Ready to submit your research? Choose BMC and benefit from:

- fast, convenient online submission

- thorough peer review by experienced researchers in your field

- rapid publication on acceptance

- support for research data, including large and complex data types

- gold Open Access which fosters wider collaboration and increased citations

- maximum visibility for your research: over 100M website views per year

At BMC, research is always in progress.

Learn more biomedcentral.com/submissions 